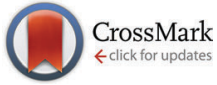

Cite this: Metallomics, 2016, 8,1170

Received 18th August 2016, Accepted 8th September 2016

DOI: $10.1039 / \mathrm{c} 6 \mathrm{mt} 00183 a$

www.rsc.org/metallomics

\section{Tuning the metabolism of the anticancer drug cisplatin with chemoprotective agents to improve its safety and efficacy}

\author{
Melani Sooriyaarachchi, ${ }^{a}$ Graham N. George, ${ }^{\text {bcd }}$ Ingrid J. Pickering, ${ }^{\text {bcd }}$ \\ Aru Narendran ${ }^{\mathrm{e}}$ and Jürgen Gailer*a
}

\begin{abstract}
Numerous in vivo studies have shown that the severe toxic side-effects of intravenously administered cisplatin can be significantly reduced by the co-administration of sulfur-containing 'chemoprotective agents'. Using a metallomics approach, a likely biochemical basis for these potentially useful observations was only recently uncovered and appears to involve the reaction of chemoprotective agents with cisplatinderived Pt-species in human plasma to form novel platinum-sulfur complexes (PSC's). We here reveal aspects of the structure of two PSC's and establish the identification of an optimal chemoprotective agent to ameliorate the toxic side-effects of cisplatin, while leaving its antineoplastic activity largely intact, as a feasible research strategy to transform cisplatin into a safer and more effective anticancer drug.
\end{abstract}

\section{Introduction}

The serendipitous discovery of the antiproliferative effects of cis-diaminedichloroplatinum(II) or cisplatin [CP] on E. coli cells in the 1960s combined with its approval by the FDA in 1978 heralded the era of platinum-based chemotherapy. ${ }^{1}$ Despite the subsequent FDA approval of second- and third-generation platinumbased anticancer drugs, such as carboplatin in 1989 and oxaliplatin in $2002,{ }^{2} \mathrm{CP}$ - which is intravenously administered either alone or in combination with other anticancer drugs - remains one of the most effective anticancer drugs that is used worldwide owing to its broad spectrum of activity towards a variety of cancers, including testicular, ovarian, head and neck, colorectal, bladder, cervical and lung cancer as well as melanoma and lymphomas. ${ }^{3}$ Extensive studies into the metabolism of $\mathrm{CP}$ have revealed that it constitutes a prodrug and it is commonly believed that the binding of the CP-derived monoaqua hydrolysis product $\left[\left(\mathrm{NH}_{3}\right)_{2} \mathrm{PtCl}\left(\mathrm{H}_{2} \mathrm{O}\right)\right]^{+}$to DNA represents its likely mode of action. ${ }^{3 c}$ Given the complexity of the intracellular biochemistry of $\mathrm{CP},{ }^{3 a, 4}$ however, further studies are needed to rule out whether other biomolecular mechanisms may also contribute to its overall activity.

\footnotetext{
${ }^{a}$ Department of Chemistry, University of Calgary, 2500 University Drive NW, Calgary, AB, T2N 1N4, Canada. E-mail: jgailer@ucalgary.ca; Fax:+403-289-9488; Tel: $+403-210-8899$

${ }^{b}$ Molecular and Environmental Science Research Group, Department of Geological Sciences, University of Saskatchewan, Saskatoon, S7N 5E2, Canada

${ }^{c}$ Toxicology Centre, University of Saskatchewan, Saskatoon, SK, S7N 5B3, Canada

${ }^{d}$ Department of Chemistry, University of Saskatchewan, Saskatoon, SK, S7N 5C9, Canada

${ }^{e}$ Division of Pediatric Oncology, Alberta Children's Hospital, Calgary, AB, T3B 6A8,

Canada
}

\section{Severe toxic side-effects of CP}

In contrast to so-called 'molecularly targeted' anticancer drugs which target a single pathway to kill cancer cells, CP represents a 'shotgun' cytotoxin which offers two major advantages: it is active against many different cell types in a tumour and its utilization is conceptually less susceptible to the development of resistance (although it does occur). ${ }^{5}$ Shotgun cytotoxins, however, also exhibit a dark side. With regard to CP, its therapeutic use and efficacy is inherently limited by the severe toxic side-effects that this metal-based drug exhibits on several non-proliferating cell types, ${ }^{4 c}$ which often results in life-long impacts on the quality of life of patients. ${ }^{6}$ For example, 30 to $60 \%$ of patients suffer from nephrotoxicity, ${ }^{7}$ more than $60 \%$ of pediatric patients develop bilateral and permanent hearing $\operatorname{loss}^{8}$ and up to $90 \%$ of patients develop neurotoxicity. ${ }^{3 b}$ Although nephrotoxicity in patients can be somewhat ameliorated by increased hydration or the administration of mannitol, ${ }^{9}$ no approved procedures exist to completely eliminate ototoxicity; the latter therefore constitutes a primary dose limiting factor. ${ }^{10}$ Likewise, there are currently no established clinical procedures to reduce or ameliorate the neurotoxicity of this otherwise very effective anticancer drug. ${ }^{11}$

\section{Strategies to ameliorate the severe toxic side-effects of Pt-based anticancer drugs}

Owing to the efficiency of $\mathrm{CP}$ and its inherent limitations, considerable research efforts are directed to improve the tumor 
selectivity of Pt-based anticancer drugs. This fundamental challenge can be addressed either by synthesizing novel Pt-compounds ${ }^{3 c, 12}$ or by improving the delivery of established or newly synthesized Pt-based drugs to the tumor by drugdelivery vehicles. ${ }^{13}$ The third principle approach - the one that is of focal interest in the context of this mini-review - aims to reduce the CP-induced severe toxic side-effects by the co-administration of small-molecular-weight 'chemoprotective agents', while leaving the anticancer effect of CP largely intact. ${ }^{14}$ Compared to the first two approaches, the latter approach is potentially more cost effective since it aims to selectively reduce the severe toxic side-effects of an already FDA-approved Pt-drug with a chemoprotective agent that may - ideally - already be approved by the FDA (i.e. costly drug approval processes are avoided altogether).

\section{Reducing $\mathrm{CP}$-induced toxic side-effects by 'chemoprotection'}

Numerous studies with animal models or patients have demonstrated that chemoprotective agents, such as sodium thiosulfate (STS) ${ }^{15} N$-acetyl-L-cysteine (NAC), ${ }^{15 a, 16}$ amifostine, ${ }^{15 c, 17}$ sodium diethyldithiocarbamate, ${ }^{10,15 c, 18}$ D-methionine, ${ }^{9,19}$ L-methionine, ${ }^{19 a, 20}$ L-glutathione $(\mathrm{GSH}),{ }^{21}$ cimetidine $^{22}$ sodium salicylate, ${ }^{23}$

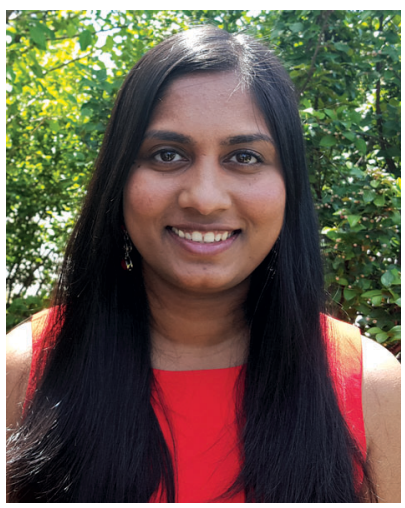

Melani Sooriyaarachchi
Melani Sooriyaarachchi was born in Sri Lanka and received her BSc (Hons) in Chemistry from the University of Colombo in 2007. She started her graduate studies at the University of Calgary in 2008 under the supervision of Dr J. Gailer where she completed her MSc (2011) and subsequently PhD (2015). Her research was focused on studying the metabolism of various clinically relevant metal-based anticancer drugs in human plasma, when used individually or in combination. She was a CIHR-THRUST fellow during 2011-2015. Currently she is a chemistry instructor at the Southern Alberta Institute of Technology.

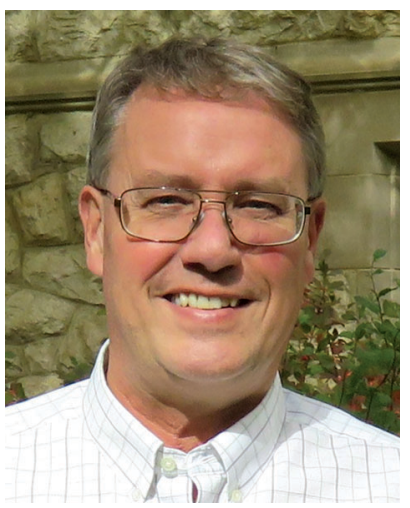

Graham N. George

Graham George was educated at King's College London (BSc, 1979) and the University of Sussex (DPhil, 1983). After postdoctoral fellowships at the University of Sussex and at Exxon Research \& Engineering Co. in New Jersey, USA, he continued at Exxon as a Principal Investigator until moving to the Stanford Synchrotron Radiation Laboratory in 1992. In 2003 took up the position of full professor and Tier 1 Canada Research Chair of X-ray Absorption Spectroscopy at the University of Saskatchewan. His research directions include a career-long interest in X-ray spectroscopy and the development of new methods for understanding the roles and mechanisms of metals in biology. He is married to fellow professor and Canada Research Chair Ingrid Pickering, and they have three children.

Aru Narendran is a full professor in Ingrid Pickering received her BA in Natural Sciences from the University of Cambridge (UK) in 1986 and following this used Physical Chemistry to study heterogeneous catalysis at the Royal Institution and received a $\mathrm{PhD}$ from Imperial College London (UK) in 1990. After two years as a postdoctoral fellow with Exxon Research and Engineering Co. (NJ, USA), she moved to the Stanford Synchrotron Radiation Laboratory (CA, USA) in 1992. She came to Canada and her present position at the University of Saskatchewan in 2003. Ingrid is currently Professor and Tier 1 Canada Research Chair in Molecular Environment Science at the University of Saskatchewan. Her research investigates the roles of metals and other elements in biological systems from the environment to human health.

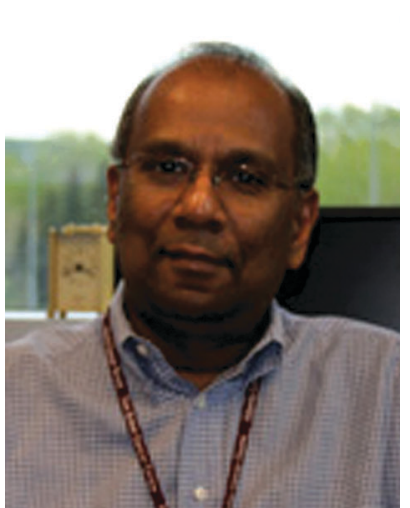

Aru Narendran the departments of pediatrics and oncology, Faculty of Medicine, University of Calgary. His primary research and clinical interests focus on the development of new agents and novel therapeutics for refractory pediatric malignancies. He is the recipient of a number of awards including the odile Schweisguth International Prize for pediatric oncology research and the young investigator award from the Children's oncology group (COG). Currently, he directs the POETIC pre-clinical and drug discovery laboratory with the mandate to identify agents and regimens for early phase clinical trials for currently difficult to treat pediatric cancer patients. 
L-carnosine $^{24}$ 2,3-dimercapto-1-propanesulfonic acid, ${ }^{25}$ and procaineamide hydrochloride ${ }^{26}$ can significantly reduce some of the severe toxic side-effects of CP. While amifostine is the only chemoprotective agent that has been specifically approved by the FDA for CP therapy, ${ }^{27}$ STS and NAC have been approved for the treatment of cyanide poisoning and for acetaminophen overdose, while D-methionine is currently undergoing a phase 3 clinical trial to reduce noise-induced hearing loss. ${ }^{6}$

From a biochemical point of view, the perhaps surprising lack of understanding as to the mode of action of the aforementioned phenomenological observations at a molecular level (i.e. the effect of chemoprotective agents on the metabolism of CP) must be attributed to the complexity of biological systems (e.g. the bloodstream) and a lack of appropriate tools to gain insight. In this context, one is reminded of F. Dyson's notion that "New directions in science are launched by new tools much more often than by new concepts. The effect of a tool driven revolution is to discover new things that need to be explained." To this end, we have developed an instrumental metallomics tool $^{28}$ that allowed us to better understand the underlying chemistry in order to ultimately translate this straightforward approach to achieve actual benefits for cancer patients. In brief, this metallomics tool allows to directly analyze blood plasma for essentially all contained Pt-species by using size-exclusion chromatography coupled on-line to an inductively coupled plasma atomic emission spectrometer. This principle approach dramatically reduces the complexity that is inherently associated with the analysis of blood plasma into all of its constituents because only the subpopulation of Pt-species is detected. It is useful to discuss some key issues that pertain to this chemoprotection approach in more detail.

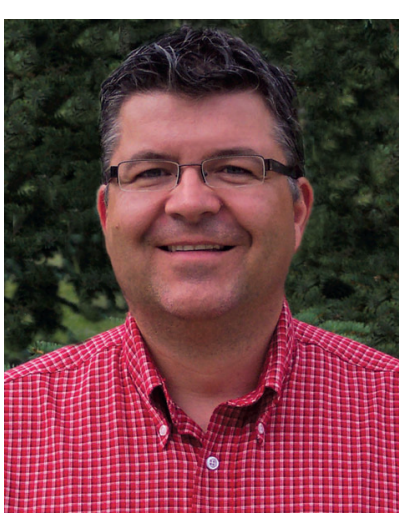

Jürgen Gailer
Jürgen Gailer received his MSc and $\mathrm{PhD}$ from the University of Graz/ Austria in 1997. As an Erwin Schroedinger fellow he moved to the Department of Molecular and Cellular Biology of the University of Arizona (Tucson/USA) and subsequently transferred to the Department of Nutritional Sciences as a research associate. In 2001 he moved to the GSF National Research Center for Environment and Health (Munich/Germany), where he was an Alexander von Humboldt fellow until 2002. In 2003 he became team leader in biopharmaceutical production at Boehringer IngelheimAustria/Vienna and joined the Department of Chemistry of the University of Calgary in 2004 where he is an associate professor and has a co-appointment in the Environmental Science Program. His research aims to develop and apply metallomics methods to better understand the role that non-essential metals and metal-based drugs play in biology.

\section{Considerations pertaining to the mechanistic aspects of the 'chemoprotection' approach}

The bloodstream represents the first biological compartment where chemical reactions between intravenously administered $\mathrm{CP}$ and a chemoprotective agent may ensue. Although relevant chemistry between CP (and its metabolites) and a chemoprotective agent may also occur in internal organs, ${ }^{29}$ it is very difficult to tune the metabolism of $\mathrm{CP}$ therein and it will therefore not be further discussed. Conceptually, the intravenous administration to a patient with an appropriate chemoprotective agent should allow one to modulate the metabolism of CP in the bloodstream, which will ultimately determine which Pt-species are left in the blood circulation to subsequently interact with healthy (unintended) and tumor tissue cells (intended) (Fig. 1). In order to evaluate the potential of this 'chemoprotection approach', one needs to first understand the metabolism of $\mathrm{CP}$ in the bloodstream itself ${ }^{28 b}$ before one can probe the effect that chemoprotective agents may exert on its metabolism (Fig. 1) as well as other blood constituents, such as erythrocytes as well as plasma proteins and metalloproteins. ${ }^{30}$

It is commonly believed that intravenously administered CP does not hydrolyze in the bloodstream, owing to the comparatively high concentration of $\mathrm{Cl}^{-}(\sim 100 \mathrm{mM})$ in human blood plasma. After the addition of CP to human plasma in vitro, however, highly toxic CP-derived hydrolysis products $(\mathrm{CPHP})^{31}$ and plasma protein bound Pt-species (Pt-PP) were present within as little as $5 \mathrm{~min},{ }^{28 b, 32}$ while the majority of Pt was still present as the parent drug. All chemoprotective agents that we have investigated - STS, NAC, D-methionine and GSH - affected the metabolism of CP in plasma by producing additional Pt-peaks that did not match the retention time of the Pt-peaks that were detected when only $\mathrm{CP}$ was added to plasma (in this case Pt-peaks corresponding to $\mathrm{CP}$,

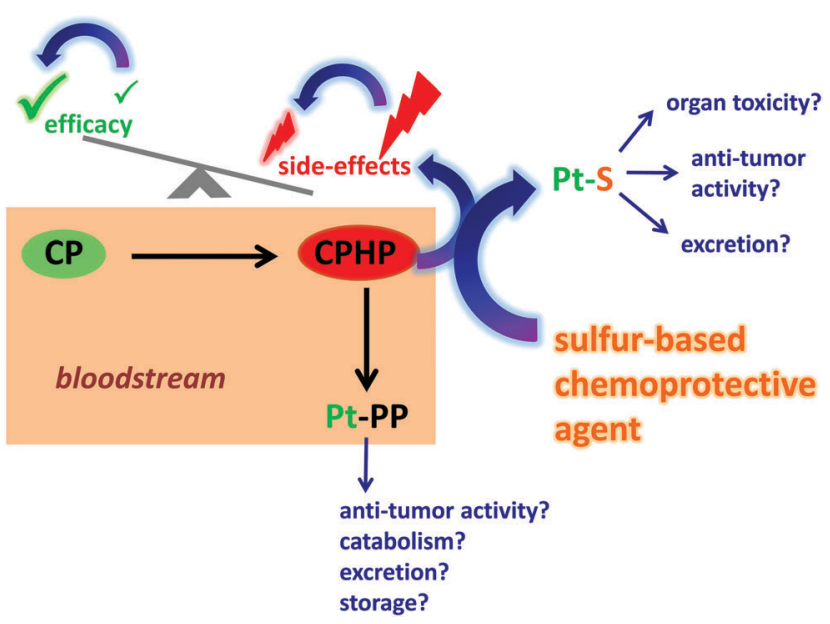

Fig. 1 Conceptual depiction of the chemical reactions that occur between cisplatin (CP) and chemoprotective agents in human plasma in vitro as well as the repercussions that these Pt-species will exert in the whole organism in vivo. CPHP refers to all CP-derived hydrolysis products and PP refers to plasma proteins. 
CPHP's and Pt-PP's were detected). ${ }^{30,33}$ The additional Pt-peaks were therefore assigned to novel Pt-containing sulfur complexes or PSC's in blood plasma. ${ }^{30,33}$ The mechanism of formation of these PSC's likely involves the reaction of the highly reactive monoaqua hydrolysis product of $\mathrm{CP}-\left[\left(\mathrm{NH}_{3}\right)_{2} \mathrm{PtCl}\left(\mathrm{H}_{2} \mathrm{O}\right)\right]^{+}$- with each chemoprotective agent. ${ }^{33 b}$ Based on this demonstrated 'tunability' of the metabolism of CP with chemoprotective agents in blood plasma, it is now possible to discuss potential advantages and disadvantages of this principle approach to possibly improve Pt-based anticancer drugs that are currently in use.

The advantages of a 'chemoprotection approach' are that the identification of an inexpensive and safe chemoprotective agent that can neutralize highly toxic CPHP's ${ }^{31}$ should allow one to reduce the severe toxic side effects of CP while minimally affecting the efficacy of the parent drug CP (Fig. 1). ${ }^{32}$ There are three potential disadvantages that have to be considered in the context of this principle approach, (a) the chemoprotective agent itself may exert adverse toxic effects by affecting the integrity of endogenous plasma metalloproteins, such as transferrin, ferritin and $\mathrm{Zn}$ bound to human serum albumin (which would result in potential toxicity), ${ }^{30}$ (b) the chemoprotective agent may decrease the plasma lifetime of the parent CP (which would decrease the efficacy of the latter) and/or (c) the PSC may not be effectively excreted, which in turn could result in possible organ toxicity (e.g. if the PSC traverses the blood-brain barrier).

\section{Tuning the metabolism of CP with an optimal chemoprotective agent}

In the context of translating this chemoprotection approach ${ }^{30,32,33}$ into practical benefits for patients (e.g. reduction of ototoxicity, neurotoxicity and/or nephrotoxicity), it is important to clearly distinguish between information that can be obtained from in vitro studies (e.g. using human plasma) and information that can only be derived from in vivo studies (e.g. using an appropriate animal model). In vitro studies are crucial to establish whether the mode of action of a chemoprotective agent involves the formation of a complex with CPHP's in plasma. If this is the case, the structure of the formed PSC can either be elucidated by X-ray absorption spectroscopy (Fig. 2) ${ }^{34}$ and/or mass spectrometry (Fig. 3). ${ }^{6}$ In vitro studies can also establish an effective molar ratio between the chemoprotective agent and CP which will preclude the formation of free CPHP's in plasma and minimize a chemoprotective agent-induced perturbation of endogenous plasma metalloproteins. ${ }^{30,33 b}$ Furthermore, the antitumour activity of the formed PSC's and their acute $\left(\mathrm{IC}_{50}\right)$ as well as their potential longterm toxicity can be established by assessing toxicogenomic endpoints ${ }^{35}$ in cell culture experiments using appropriate cell lines. After the completion of these in vitro studies, in vivo studies with an appropriate animal model are absolutely necessary to further optimize the molar ratio between the chemoprotective agent and CP, their order of injection as well as the time lag between the administration of the two drugs. ${ }^{36}$ In addition, in vivo studies are required to detect a potentially adverse organ accumulation of a formed PSC (e.g. in the brain) and to assess the clearance of formed PSC complexes from the bloodstream to the kidneys and ultimately the urine.

\section{Future outlook}

The elucidation of the mechanisms by which chemoprotective agents affect the metabolism of CP in the bloodstream is absolutely critical in the context of developing a clinical treatment protocol that can be employed to significantly reduce the toxic side-effects of CP in patients while maintaining its antitumor efficacy. This task is now within reach since state-of-the-art metallomics tools can be applied in conjunction with animal studies. ${ }^{30,33 a, b}$ In principle, the approach of selectively inactivating the toxic hydrolysis products of $\mathrm{CP}$ in the bloodstream by an optimal chemoprotective agent, while maximizing the lifetime of the active anticancer prodrug $\mathrm{CP}$ in the blood circulation would effectively transform this anticancer drug into a much safer one.
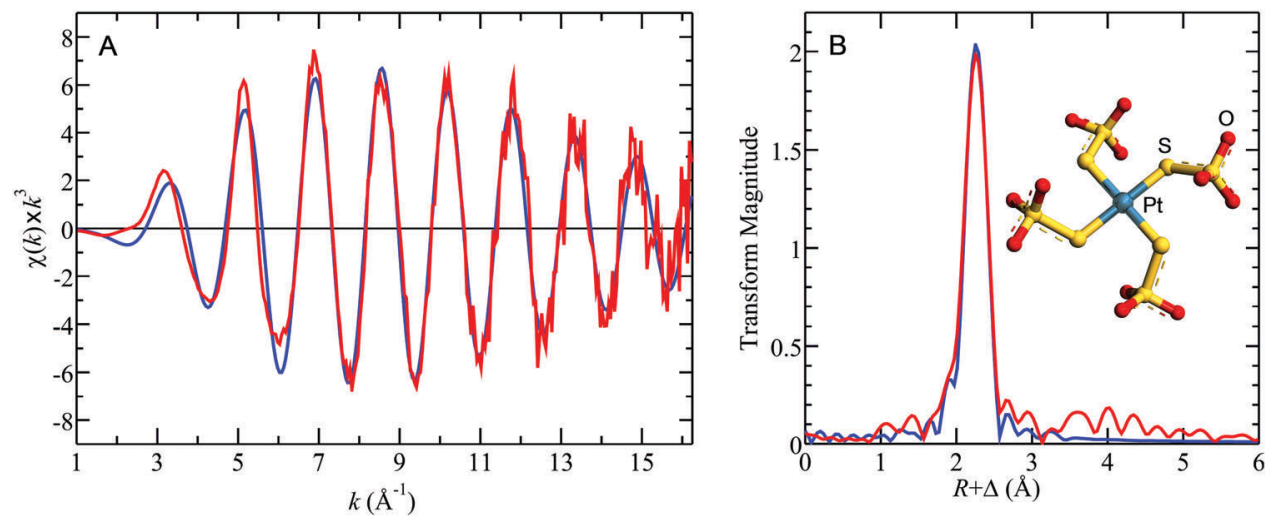

Fig. 2 X-ray absorption spectroscopy-derived structure of the $\left[\mathrm{Pt}\left(\mathrm{S}_{2} \mathrm{O}_{3}\right)_{4}\right]^{6-}$ that was isolated from PBS-buffer (STS: CP = 400:1). (A) Shows the Pt $\mathrm{L}_{\text {III }}$ EXAFS oscillations and (B) the Pt-S phase corrected Fourier transforms, for data (red lines), together with the best fit (blue lines). Best fits indicated four equivalent $\mathrm{Pt}-\mathrm{S}$ at distance $R$ of $2.31 \AA$, with $\sigma^{2}$, the mean square deviation in $R$, of $0.0031 \AA^{2}$. The inset shows the energy minimized geometry optimized density functional theory structure for $\left[\mathrm{Pt}\left(\mathrm{S}_{2} \mathrm{O}_{3}\right)_{4}\right]^{6-}$ constrained to $\mathrm{S}_{4}$ point group symmetry to assist with convergence. The computed Pt-S bondlengths were $2.33 \AA$, in excellent agreement with the EXAFS results, and both are in agreement with the Pt-S distance of the sole reported crystal structure of a terminal thiosulfate bound to a Pt(II) ion. ${ }^{34}$ 


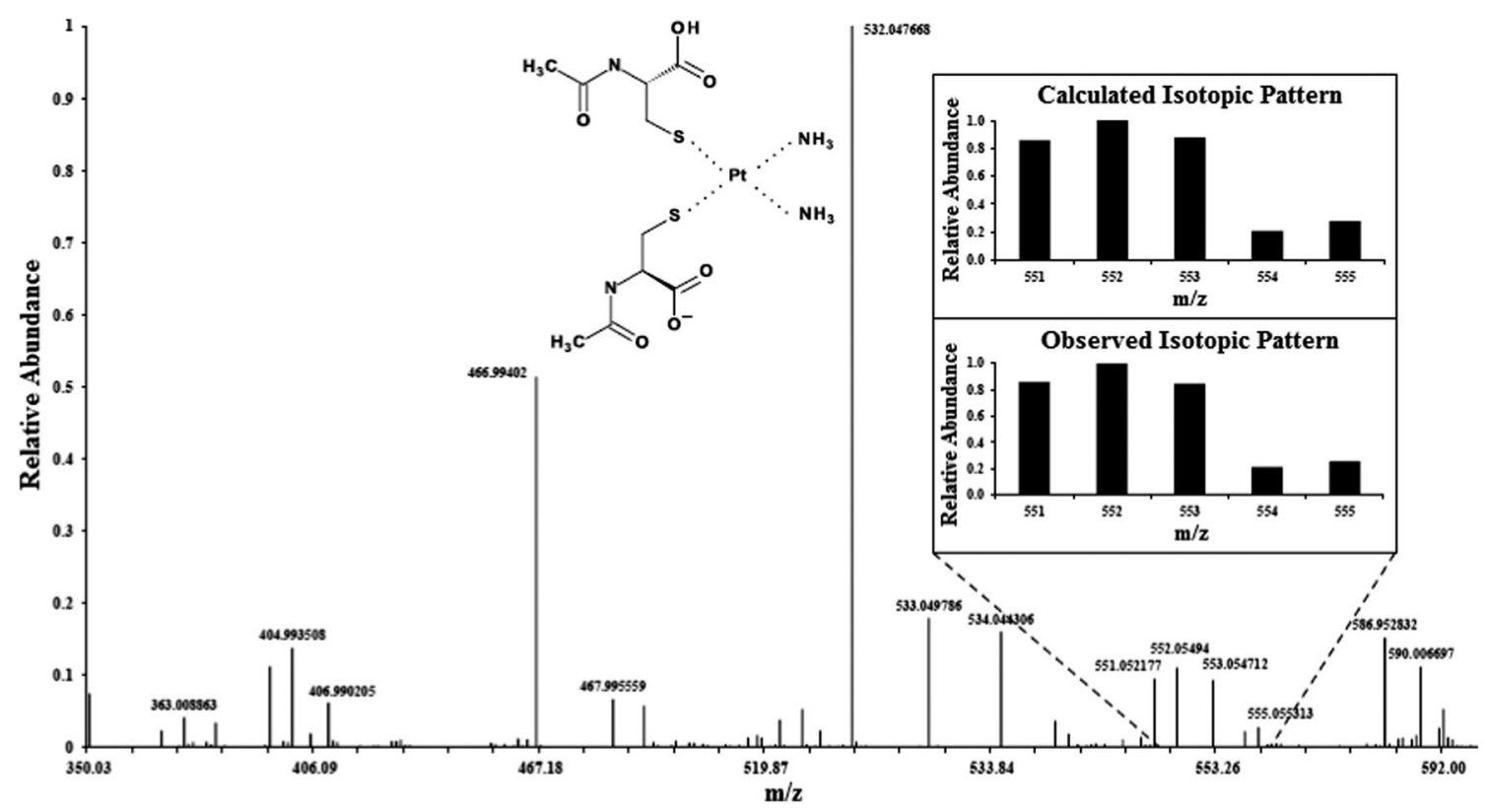

Fig. 3 A LC-ESI-MS spectrum (negative mode) obtained when PBS-buffer was spiked with $\mathrm{CP}$, incubated at $37^{\circ} \mathrm{C}$ for 10 min and then spiked with NAC and analyzed 50 min later. ${ }^{6}$ NAC: CP molar ratio $400: 1, C P$ : cisplatin, NAC: $N$-acetylcysteine.

This overall strategy may also allow one to improve the therapeutic potential of CP (as well as other toxic metal-based anticancer drugs) by escalating the dose that is administered to a patient. This would be particularly useful as this would allow one to treat even those patients in which the tumor has developed drug resistance and where the $\mathrm{CP}$ dose can often not be further increased because of the inherent severe toxic side-effects of CP.

\section{Acknowledgements}

MS is a Fellow in the Canadian Institutes of Health Research Training grant in Health Research Using Synchrotron Techniques (CIHR-THRUST). AN is supported by a research grant from the Alberta's Children's Hospital Foundation. GNG and IJP are supported by Canada Research Chairs, with research at the University of Saskatchewan further supported by NSERC, CIHR, SHRF and the Government of Saskatchewan. Use of the Stanford Synchrotron Radiation Lightsource (SSRL), SLAC National Accelerator Laboratory is supported by the U.S. DOE, Office of Science, OBES under Contract No. DE-AC02-76SF00515. The SSRL Structural Molecular Biology Program is supported by the DOE Office of Biological and Environmental Research and by the National Institutes of Health (NIH), National Institute of General Medical Sciences (NIGMS) including P41GM103393. The contents of this publication are solely the responsibility of the authors and do not necessarily represent the official views of NIGMS or NIH.

\section{References}

1 A. V. Klein and T. W. Hambley, in Ligand Design in Medicinal Inorganic Chemistry, ed. T. Storr, John Wiley \& Sons, Ltd, 1st edn, 2014, pp. 9-45.
2 L. Kelland, The resurgence of platinum-based cancer chemotherapy, Nat. Rev. Cancer, 2007, 7, 573-584.

3 (a) C.-P. Tan, Y.-Y. Lu, L.-N. Ji and Z.-W. Mao, Metallomics insights into the programmed cell death induced by metalbased anticancer drugs, Metallomics, 2014, 6, 978-995; (b) A. Maccio and C. Madeddu, Cisplatin: an old drug with a newfound efficacy - from mechanisms of action to cytotoxicity, Expert Opin. Pharmacother., 2013, 14, 1839-1857; (c) A. M. Pizarro and P. J. Sadler, Unusual DNA binding modes for metal anticancer complexes, Biochimie, 2009, 91, 1198-1211.

4 (a) D. Gibson, The mechanism of action of platinum anticancer drugs-what do we really know about it, Dalton Trans., 2009, 10681-10689; (b) A. Casini and J. Reedijk, Interactions of anticancer Pt compounds with proteins: an overlooked topic in medicinal inorganic chemistry, Chem. Sci., 2012, 3, 3135-3144; (c) S. M. Sancho-Martinez, L. Prieto-Garcia, M. Prieto, J. M. Lopez-Novoa and F. J. Lopez-Hernandez, Subcellular targets of cisplatin cytotoxicity: an integrated view, Pharmacol. Ther., 2012, 136, 35-55.

5 T. W. Hambley, Developing new metal-based therapeutics: challenges and opportunities, Dalton Trans., 2007, 4929-4937.

6 M. Sooriyaarachchi, Ameliorating the toxic side-effects of cisplatin by systematically modulating its metabolism in human plasma with chemoprotective agents, $\mathrm{PhD}$ thesis, University of Calgary, April 2015.

7 (a) N. Pabla and Z. Dong, Cisplatin nephrotoxicity: mechanisms and renoprotective strategies, Kidney Int., 2008, 73, 994-1007; (b) V. Sahni, D. Choudhury and Z. Ahmed, Chemotherapyassociated renal dysfunction, Nat. Rev. Nephrol., 2009, 5, 450-462.

8 P. R. Brock, K. R. Knight, D. R. Freyer, K. C. M. Campbell, P. S. Steyger, B. W. Blakely, S. R. Rassekh, K. W. Chang, B. J. Fliogor, K. Rajput, M. Sullivan and E. A. Neuwelt, 
Platinum-induced ototoxicity in children: a consensus review on mechanisms, predisposition, and protection, including a new international society of pediatric oncology Boston ototoxicity scale, J. Clin. Oncol., 2012, 30, 2408-2417.

9 K. C. M. Campbell, L. P. Rybak, R. P. Meech and L. Hughes, D-Methionine provides excellent protection from cisplatin ototoxicity in the rat, Hear. Res., 1996, 102, 90-98.

10 J. M. Berry, C. Jacobs, B. I. Sikic, J. Halsey and R. F. Borch, Modification of cisplatin toxicity with diethyldithiocarbamate, J. Clin. Oncol., 1990, 8, 1585-1590.

11 S. Wolf, D. Barton, L. Kottschade, A. Grothey and C. Loprinzi, Chemotherapy-induced peripheral neuropathy: prevention and treament strategies, Eur. J. Cancer, 2008, 44, 1507-1515.

12 (a) B. W. Harper, A. M. Krause-Heuer, M. P. Grant, M. Manohar, K. B. Garbutcheon-Singh and J. R. AldrichWright, Advances in platinum chemotherapeutics, Chem. Eur. J., 2010, 16, 7064-7077; (b) P. C. A. Bruijnincx and P. J. Sadler, New trends for metal complexes with anticancer activity, Curr. Opin. Chem. Biol., 2008, 12, 197-206.

13 (a) L. Liao, J. Liu, E. C. Dreaden, S. W. Morton, K. E. Shopsowitz, P. T. Hammond and J. A. Johnson, A convergent synthetic platform for single-nanoparticle combination cancer therapy: ratiometric loading and controlled release of cisplatin, doxorubicin, and camptothecin, J. Am. Chem. Soc., 2014, 136, 5896-5899; (b) A. M. Krause-Heuer, N. J. Wheate, M. J. Tilby, D. G. Pearson, C. J. Ottley and J. R. AldrichWright, Substituted $b$-cyclodextrin and calix[4]arene as encapsulatory vehicles for platinum(II)-based DNA intercalators, Inorg. Chem., 2008, 47, 6880-6888; (c) F. Kratz, I. A. Muller, C. Ryppa and A. Warnecke, Prodrug strategies in anticancer chemotherapy, ChemMedChem, 2008, 3, 20-53; (d) J. A. Plumb, B. Venugopal, R. Oun, N. Gomez-Roman, Y. Kawazoe, N. S. Venkataramanan and N. J. Wheate, Curcubit[7]uril encapsulated cisplatin overcomes cisplatin resistance via a pharmacokinetic effect, Metallomics, 2012, 4, 561-567.

$14 \mathrm{X}$. Wang and Z. Guo, The role of sulfur in platinum anticancer chemotherapy, Anti-Cancer Agents Med. Chem., 2007, 7, 19-34.

15 (a) D. T. Dickey, Y. J. Wu, L. L. Muldoon and E. A. Neuwelt, Protection against cisplatin-induced toxicities by $\mathrm{N}$-acetylcysteine and sodium thiosulfate as assessed at the molecular, cellular, and in vivo levels, J. Pharmacol. Exp. Ther., 2005, 314, 1052-1058; (b) T. Saito, Z. J. Zhang, Y. Manabe, T. Ohtsubo and $\mathrm{H}$. Saito, The effect of sodium thiosulfate on ototoxicity and pharmacokinetics after cisplatin treatment in guinea pigs, Eur. Arch. Oto-Rhino-Laryngol., 1997, 254, 281-286; (c) D. R. Gandara, V. J. Wiebe, E. A. Perez, R. W. Makuch and M. W. deGregorio, Cisplatin rescue therapy: experience with sodium thiosulfate, WR2721, and diethyldithiocarbamate, Crit. Rev. Oncol. Hematol., 1990, 10, 353-365; (d) B. Erdlenbruch, A. Pekrun, H. Schiffman, O. Witt and M. Lakomek, Accidental cisplatin overdose in a child: reversal of acute renal failure with sodium thiosulfate, Med. Pediatr. Oncol., 2002, 38, 349-352; (e) N. Nagai, K. Hotta, H. Yamamura and H. Ogata, Effects of sodium thiosulfate on the pharmacokinetics of unchanged cisplatin and on the distribution of platinum species in rat kidney: protective mechanism against cisplatin nephrotoxicity, Cancer Chemother. Pharmacol., 1995, 36, 404-410.

16 (a) L. L. Muldoon, S. L. Walker-Rosenfeld, C. Halce, S. E. Purcell, L. C. Bennett and E. A. Neuwelt, Rescue from enhanced alkylator-induced cell death with low molecular weight sulfur-containing chemoprotectants, J. Pharmacol. Exp. Ther., 2001, 296, 797-805; (b) Y. J. Wu, L. L. Muldoon and E. A. Neuwelt, The chemoprotective agent $\mathrm{N}$-acetylcysteine blocks cisplatin-induced apoptosis through caspase signaling pathway, J. Pharmacol. Exp. Ther., 2005, 312, 424-431; (c) D. T. Dickey, L. L. Muldoon, N. D. Doolittle, D. R. Peterson, D. F. Kraemer and E. A. Neuwelt, Effect of N-acetylcysteine route of administration on chemoprotection against cisplatininduced toxicity in rat models, Cancer Chemother. Pharmacol., 2008, 62, 235-241.

17 (a) M. Treskes and W. J. F. van der Vijgh, WR2721 as a modulator of cisplatin- and carboplatin-induced side effects in comparison with other chemoprotective agents: a molecular approach, Cancer Chemother. Pharmacol., 1993, 33, 93-106; (b) D. Glover, S. Grabelsky, K. Fox, C. Weiler, L. Cannon and J. Glick, Clinical trials of WR-2721 and cis-platinum, Int. J. Radiat. Oncol., Biol., Phys., 1989, 16, 1201-1204.

18 (a) L. P. Rybak, R. Ravi and S. M. Somani, Mechanism of protection by diethyldithiocarbamate against cisplatin ototoxicity: antioxidant system, Toxicol. Sci., 1995, 26, 293-300; (b) R. F. Borch and M. E. Pleasants, Inhibition of cis-platinum nephrotoxicity by diethyldithiocarbamate rescue in a rat model, Proc. Natl. Acad. Sci. U. S. A., 1979, 76, 6611-6614; (c) M. W. Church, J. A. Kaltenbach, B. W. Blakely and D. L. Burgio, The comparative effects of sodium thiosulfate, diethyldithiocarbamate, fosfomycin and WR-2721 on ameliorating cisplatin induced ototoxicity, Hear. Res., 1995, 86, 195-203.

19 (a) M. M. Jones and M. Basinger, Thiol and thioether suppression of cis-platinum-induced nephrotoxicity in rats bearing the Walker 256 carcinosarcoma, Anticancer Res., 1989, 9, 1937-1942; (b) N. G. Cloven, A. Re, M. T. McHale, R. A. Burger, P. J. Disaia, G. S. Rose, K. C. M. Campbell and H. Fan, Evaluation of D-methionine as a cytoprotectant in cisplatin treatment of an animal model for ovarian cancer, Anticancer Res., 2000, 20, 4205-4210; (c) A. Ekborn, G. Laurell, P. Johnström, I. Wallin, S. Eksborg and H. Ehrsson, D-Methionine and cisplatin ototoxicity in the guinea pig: D-methionine influences cisplatin pharmacokinetics, Hear. Res., 2002, 165, 53-61; (d) K. C. M. Campbell, R. P. Meech, L. P. Rybak and L. F. Hughes, D-Methionine protects against cisplatin damage to the stria vascularis, Hear. Res., 1999, 138, 13-28; (e) K. D. Korver, L. P. Rybak, C. Whitworth and K. M. Campbell, Round window application of D-methionine provides complete cisplatin otoprotection, Otolaryngol.Head Neck Surg., 2002, 126, 683-689.

20 M. A. Basinger, M. M. Jones and M. A. Holscher, L-Methionine antagonism of cis-platinum nephrotoxicity, Toxicol. Appl. Pharmacol., 1990, 103, 1-15.

21 (a) F. Hamers, J. H. Brakkee, E. Cavalletti, M. Tedeschi, L. Marmonti, G. Pezzoni, J. P. Neijt and W. H. Gispen, 
Reduced glutathione protects against cisplatin-induced neurotoxicity in rats, Cancer Res., 1993, 53, 544-549; (b) F. Zunino, G. Pratesi, A. Micheloni, E. Cavalletti, F. Sala and O. Tofanetti, Protective effect of reduced glutathione against cisplatin-induced renal and systemic toxicity and its influence on the therapeutic activity of the antitumor drug, Chem.-Biol. Interact., 1989, 70, 89-101; (c) F. Re, S. Bohm, S. Oriana, G. Battista Spatti and F. Zunino, Efficacy and safety of high-dose cisplatin and cyclophosphamide with glutathione protection in the treatment of bulky advanced epithelial ovarian cancer, Cancer Chemother. Pharmacol., 1990, 25, 355-360.

22 H. Katsuda, M. Yamashita, H. Katsura, J. Yu, Y. Waki, N. Nagata, Y. Sai and K. Miyamoto, Protecting cisplatininduced nephrotoxicity with cimetidine does not affect antitumor activity, Biol. Pharm. Bull., 2010, 33, 1867-1871.

23 (a) M. A. Hyppolito, J. A. A. de Oliveira and M. Rossato, Cisplatin ototoxicity and otoprotection with sodium salicylate, Eur. Arch. Oto-Rhino-Laryngol., 2006, 263, 798-803; (b) G. M. Li, S. H. Sha, E. Zotova, J. Arezzo, T. Van de Water and J. Schacht, Salicylate protects hearing and kidney function from cisplatin toxicity without compromising its oncolytic action, Lab. Invest., 2002, 82, 585-596.

24 A. A. Fouad, M. A. Morsy and W. Gomaa, Protective effect of carnosine against cisplatin-induced nephrotoxicity in mice, Environ. Toxicol. Pharmacol., 2008, 25, 292-297.

25 T. Sato, M. Okubo, K. Sawaki, H. Maehashi and M. Kawaguchi, Paradoxical effect of 2,3-dimercapto-1-propanesulfonic acid (DMPS) on enhancing antitumor activity of cisplatin in ascites sarcoma 180 cells, J. Pharmacol. Sci., 2010, 112, 361-368.

26 (a) M. Viale, J. X. Zhang, M. Pastrone, M. A. Mariggio, M. Esposito and W. E. Lindup, Reduction of cisplatin nephrotoxicity by procaineamide: does the formation of a cisplatin-procaineamide complex play a role?, J. Pharmacol. Exp. Ther., 2000, 293, 829-836; (b) E. Ognio, B. Chiavarina, M. Peterka, M. A. Mariggio and M. Viale, Study of feasibility of the treatment with procaineamide hydrochloride and cisplatin in pregnant mice, Chem.-Biol. Interact., 2006, 164, 232-240; (c) A. Zicca, S. Cafaggi, M. A. Mariggi, M. O. Vannozzi, V. Ottone, V. Bocchini, G. Caviglioli and M. Viale, Reduction of cisplatin hepatotoxicity by procaineamide hydrochloride in rats, Eur. J. Pharmacol., 2002, 442, 265-272; (d) C. Fenoglio, E. Boncompagni, B. Chiavarina, S. Cafaggi, M. Cilli and M. Viale, Morphological and histochemical evidence of the protective effect of procaineamide hydrochloride on tissue damage induced by repeated administration of low doses of cisplatin, Anticancer Res., 2005, 25, 4123-4128.

27 A. Gula, L. Ren, Z. Zhou, D. Lu and S. Wang, Design and evaluation of biodegradable enteric microcapsules of amifostine for oral delivery, Int. J. Pharmacol., 2013, 453, 441-447.
28 (a) S. A. Manley, S. Byrns, A. W. Lyon, P. Brown and J. Gailer, Simultaneous $\mathrm{Cu}-, \mathrm{Fe}-$, and $\mathrm{Zn}$-specific detection of metalloproteins contained in rabbit plasma by size-exclusion chromatography-inductively coupled plasma atomic emission spectroscopy, J. Biol. Inorg. Chem., 2009, 14, 61-74; (b) M. Sooriyaarachchi, A. Narendran and J. Gailer, Comparative hydrolysis and plasma binding of cis-platin and carboplatin in human plasma in vitro, Metallomics, 2011, 3, 49-55.

29 N. C. Schmitt, E. W. Rubel and N. M. Nathanson, Cisplatininduced hair cell death requires STAT1 and is attenuated by epigallocatechin gallate, J. Neurosci., 2009, 29, 3843-3851.

$30 \mathrm{M}$. Sooriyaarachchi, A. Narendran and J. Gailer, N-Acetyl-Lcysteine modulates the metabolism of cis-platin in human plasma in vitro, Metallomics, 2013, 5, 197-207.

31 P. T. Daley-Yates and D. C. H. McBrien, Cisplatin metabolites in plasma, study of their pharmacokinetics and importance in the nephrotoxic and antitumor activity of cisplatin, Biochem. Pharmacol., 1984, 33, 3063-3070.

32 T. T. Morris, Y. Ruan, V. A. Lewis, A. Narendran and J. Gailer, Fortification of blood plasma from cancer patients with human serum albumin decreases the concentration of cisplatin-derived hydrolysis products in vitro, Metallomics, 2014, 6, 2034-2041.

33 (a) M. Sooriyaarachchi, A. Narendran and J. Gailer, The effect of sodium thiosulfate on the metabolism of cis-platin in human plasma in vitro, Metallomics, 2012, 4, 960-967; (b) M. Sooriyaarachchi, A. Narendran, W. H. White and J. Gailer, Chemoprotection by D-methionine against cis-platininduced side-effects: insight from in vitro studies using human plasma, Metallomics, 2014, 6, 532-541; (c) M. Sooriyaarachchi, M. A. Gibson, B. d. S. Lima and J. Gailer, Modulation of the metabolism of cis-platin in blood plasma by glutathione, Can. J. Chem., 2016, 94, 360-366.

34 M. Sooriyaarachchi, J. Gailer, N. V. Dolgova, I. J. Pickering and G. N. George, Chemical basis for the detoxification of cisplatin-derived hydrolysis products by sodium thiosulfate, J. Inorg. Biochem., 2016, DOI: 10.1016/j.jinorgbio.2016.06.012.

35 H. Ellinger-Ziegelbauer, J. M. Fostel, C. Aruga, D. Bauer, S. Deng, D. Dickinson, A. C. Le Fevre, A. J. J. Fornace, Y. Gu, J. C. Hoflack, M. Shiiyama, R. Smith, R. D. Snyder, C. Spire, G. Tanaka and J. Aubrecht, Characterization and interlaboratory comparison of a gene expression signature for differentiating genotoxic mechanisms, Toxicol. Sci., 2009, 110, 341-352.

36 L. L. Muldoon, M. A. Pagel, R. A. Kroll, R. E. Brummett, N. D. Doolittle, E. G. Zuhowski, M. J. Egorin and E. A. Neuwelt, Delayed administration of sodium thiosulfate in animal models reduces platinum ototoxicity without reduction of antitumor activity, Clin. Cancer Res., 2000, 6, 309-315. 